\title{
XIII. Equipamiento en ventilación no invasiva
}

\author{
ORLANDO DÍAZ P.*
}

\section{Equipment for noninvasive ventilation}

Ventilación no invasiva (VNI) es la aplicación de ventilación mecánica sin intubación endotraqueal. Su empleo forma parte del tratamiento estándar de una serie de condiciones clínicas que cursan con insuficiencia respiratoria aguda o crónica. La expansión de sus indicaciones dentro y fuera del hospital, ha hecho necesario que el personal de salud involucrado directa o indirectamente con su uso adquiera conocimientos del equipamiento requerido para su aplicación. Esta tarea no siempre es fácil, teniendo en cuenta los crecientes avances tecnológicos, pero es indispensable ya que el éxito de la VNI depende de una apropiada selección y empleo de los diferentes ventiladores e interfaces.

\section{Ventiladores convencionales $\mathbf{y}$ portátiles}

Para aplicar la VNI se utilizan ventiladores de presión positiva. Estos son de dos tipos: los ventiladores convencionales y los equipos portátiles para ventilación no invasiva. Los ventiladores convencionales son aquellos utilizados en las unidades de cuidados intensivos (UCI) y se caracterizan por poseer una gran variedad de modos ventilatorios, monitorización completa y un amplio sistema de alarmas. Los ventiladores portátiles para ventilación no invasiva son equipos que proporcionan flujo continuo, permiten aplicar presión positiva continua de la vía aérea (CPAP; continuous positive airway pressure) o ventilación con presión de dos niveles, por lo que también se les denomina ventiladores de presión binivel (BiPAP); excepcionalmente poseen sistemas de monitorización, cuentan con un número limitado de alarmas y están diseñados específicamente para proporcionar ventilación no invasiva. La mayoría de los ensayos clínicos en que se ha empleado ventilación no invasiva en pacientes con insuficiencia respiratoria aguda han empleado ventiladores portátiles ${ }^{1}$.

Ventiladores convencionales. Estos ventiladores tienen la capacidad de proporcionar una $\mathrm{FIO}_{2}$ regulable entre 21 y $100 \%$, permitir monitorización completa mediante curvas de presión, flujo y volumen, y disponer de un sistema completo de alarmas. Asimismo, su gama de modos ventilatorios es amplia y permite aplicar CPAP, modalidades controladas por volumen y por presión. Una de sus grandes desventajas es la limitada capacidad para compensar fugas, problema no menor, ya que las fugas son muy frecuentes cuando se emplea VNI. Esto se ha solucionado en algunos ventiladores más recientes, que han sido adaptados para proporcionar ventilación no invasiva, como es el caso del Dragger Evita 4, Evita XL y Savina; el Maquet Servo-i; el Puritan Bennett 840; los equipos Viasys AVEA, Vela y LTV 1150; y el Newport e360. No se sabe con certeza si su capacidad de compensación de fugas es comparable a la de los equipos portáti$l^{2}{ }^{2}$. En aquellos que no poseen esta capacidad, la VNI debe aplicarse con interfaces que aseguren una gran adherencia al rostro del paciente y minimicen las fugas.

La asistencia ventilatoria no invasiva con estos ventiladores puede efectuarse con modalidades controladas por presión o volumen (ver Modalidades). Aunque no se ha demostrado diferencias fisiológicas entre ambos modos $^{3}$, la ventilación controlada por presión como es el caso de la presión de soporte (PSV) es mejor tolerada que la controlada por volumen.

Ventiladores portátiles de flujo continuo. Estos equipos están diseñados exclusivamente para proporcionar ventilación no invasiva. Poseen un

Profesor Asociado de Medicina, Departamento de Enfermedades Respiratorias, Pontificia Universidad Católica de Chile. 
sistema de inyección continuo de gas operado por una turbina y limitado por una válvula solenoide que regula el flujo (Figura 1).

Los niveles de flujo son controlados por un microprocesador, retroalimentado por un neumotacógrafo, y generan un flujo que es continuo pero cuya magnitud varía cíclicamente. Estas variaciones se traducen en una presión positiva inspiratoria y una espiratoria (de allí el nombre de presión binivel), que son reguladas por el operador. Las presiones se denominan, por sus siglas en inglés, IPAP (inspiratory positive airway pressure) y EPAP (expiratory positive airway pressure). La modalidad binivel es equivalente a la PSV + PEEP de los equipos convencionales. La única diferencia reside en la programación del ventilador; en los equipos convencionales, se programa separadamente el soporte inspiratorio y la presión positiva de final de espiración (PEEP). En los equipos portátiles, el soporte inspiratorio corresponde a la diferencia entre IPAP y EPAP. En otras palabras, una IPAP/EPAP de $15 / 5 \mathrm{~cm} \mathrm{H}_{2} \mathrm{O}$ en el equipo portátil, corresponde a una PSV de $10 \mathrm{H}_{2} \mathrm{O}$ y una PEEP de $5 \mathrm{~cm} \mathrm{H}_{2} \mathrm{O}$ en el ventilador convencional.

Los ventiladores portátiles utilizan un circuito único sin válvula espiratoria verdadera. El gas espirado sale al ambiente a través de pequeños orificios o ventanas localizados en la interface o en el mismo circuito cerca de la interface. Estos orificios se denominan puertos de fuga intencional y serán mencionados más adelante.

La particularidad de generar un flujo continuo y la velocidad de respuesta de la válvula solenoi- de y la turbina frente a aumentos de la demanda de flujo son las características que les permiten a los equipos portátiles compensar las fugas del sistema ${ }^{4-6}$.

Además de la ausencia de alarmas y monitorización, existen dos problemas específicos de los ventiladores de flujo continuo. Estos son la reinhalación de $\mathrm{CO}_{2}$ y la incapacidad para administrar oxígeno suplementario en forma controlada.

Reinhalación de $\mathrm{CO}_{2}$. Los ventiladores convencionales no mezclan los gases inspirados y espirados, ya que poseen doble tubería. La tubería única de los ventiladores portátiles tiene el riesgo potencial de provocar la reinhalación del $\mathrm{CO}_{2}$ del gas espirado. Las válvulas de no-reinhalación permiten una efectiva remoción de $\mathrm{CO}_{2}{ }^{7}$, pero incrementan la resistencia espiratoria y pueden aumentar el trabajo respiratorio ${ }^{8}$. Afortunadamente el uso de tales válvulas no es indispensable. Si el ventilador mantiene un flujo continuo lo suficientemente elevado, impide el movimiento retrógrado del gas exhalado hacia el circuito durante la espiración. Para lograrlo se requiere utilizar niveles de EPAP entre $4-6 \mathrm{~cm}$ $\mathrm{H}_{2} \mathrm{O}^{9}$. Así se obtiene la adecuada salida del gas espirado hacia el ambiente por los orificios o ventanas de fuga intencional ya mencionados. Al ser ésta una fuga que el ventilador puede "reconocer", es compensada y no incide en su funcionamiento.

Para un correcto desempeño, la mejor posición de estos orificios es en la interface, sobre el puente nasal ${ }^{9}$, aunque pueden obtenerse resultados semejantes cuando están ubicados en otras

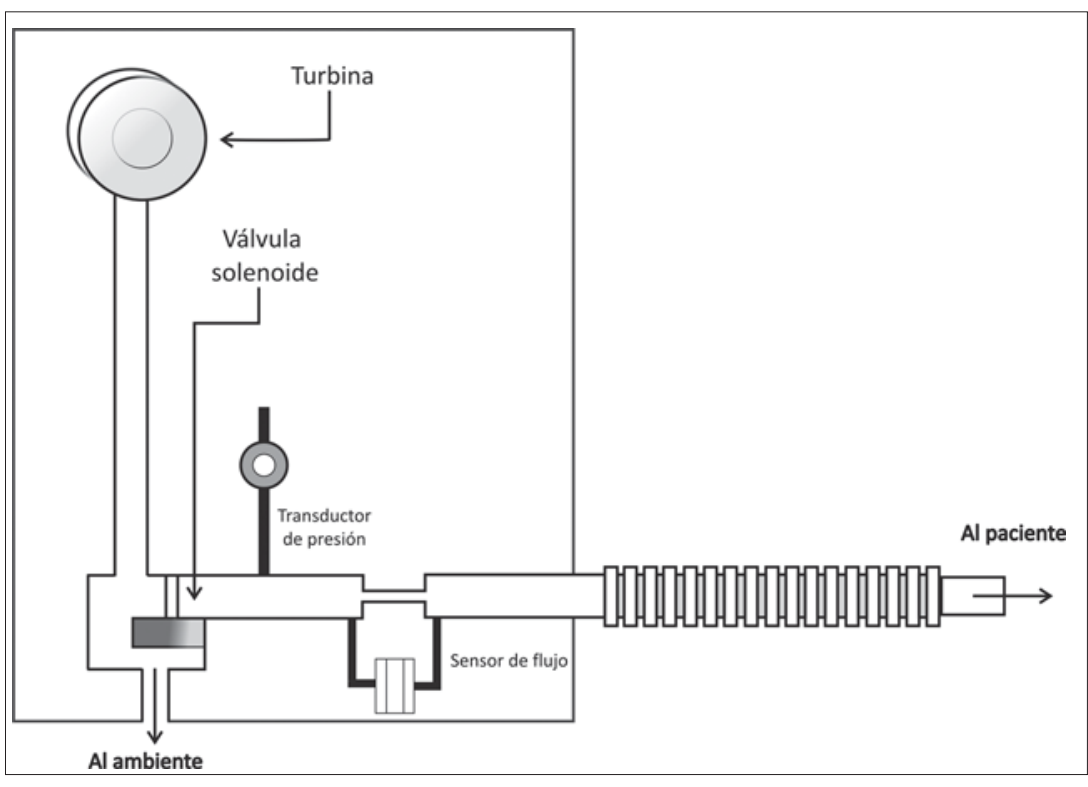

Figura 1. Diagrama de un ventilador portátil de flujo continuo. El flujo administrado depende de la velocidad a que gire la turbina y está en directa relación con la presión inspiratoria seleccionada (IPAP, inspiratory positive airway pressure). El flujo que llega al paciente es regulado por una válvula solenoide. Cuando el ventilador cicla a EPAP (expiratory positive airway pressure), la válvula se abre al ambiente y el flujo por el circuito hacia el paciente disminuye. En IPAP, la válvula se encuentra casi completamente cerrada y el flujo de la turbina pasa hacia el paciente. 
posiciones de la interface. En otras palabras, la condición ideal es que estos orificios espiratorios se encuentren en una posición de la interface que sea opuesta a la entrada del circuito inspiratorio. Aunque no se ha demostrado que la reinhalación de $\mathrm{CO}_{2}$ tenga importancia clínica ${ }^{10}$, se recomienda tomar las precauciones señaladas cuando la VNI se aplica a pacientes hipercápnicos.

Oxígeno suplementario. Los ventiladores convencionales pueden proporcionar $\mathrm{FIO}_{2}$ entre 21 y $100 \%$, porque cuentan con mezcladores. La mayoría de los ventiladores portátiles utiliza aire ambiental, de manera que para aumentar la $\mathrm{FIO}_{2}$ debe agregarse oxígeno suplementario. Generalmente el oxígeno puede adicionarse usando una conexión a la salida del ventilador o usando un puerto $a d-h o c$ en la misma interface. Si bien se ha observado que al agregarlo en un punto intermedio del circuito se logra una mayor $\mathrm{FIO}_{2}$, esto resulta imposible de hacer en los circuitos convencionales ${ }^{11}$.

La $\mathrm{FIO}_{2}$ obtenida depende de tres factores: a) el punto donde el oxígeno se agrega al circuito y la localización del orificio de fuga; b) el nivel de IPAP/EPAP; y c) el flujo de oxígeno. Si el oxígeno se agrega al circuito, la $\mathrm{FIO}_{2}$ es mayor cuando la fuga está localizada en la interface; en cambio, si el oxígeno se agrega en la interface la $\mathrm{FIO}_{2}$ es mayor si el orificio de fuga está en el circuito ${ }^{12}$. A su vez, cuando la presión de soporte (es decir, IPAP-EPAP) es mayor a $10 \mathrm{~cm} \mathrm{H}_{2} \mathrm{O}$, se requieren flujos de $\mathrm{O}_{2}$ entre 10$15 \mathrm{~L} / \mathrm{min}$ para lograr $\mathrm{FIO}_{2} \sim 50 \%{ }^{11,12}$. Los analizadores de oxígeno insertados en el circuito del ventilador portátil o en la interface proporcionan información poco confiable, de manera que es imposible conocer la $\mathrm{FIO}_{2}$ real. Por ello, la administración de $\mathrm{O}_{2}$ suplementario debe guiarse más bien por la $\mathrm{SpO}_{2}$. Si la condición clínica requiere el empleo de concentraciones de oxígeno mayor de $50 \%$ y estables es necesario un ventilador con mezclador, lo que en la actualidad sólo está disponible en algunos ventiladores de flujo continuo, como es el caso del modelo VISION® (Respironics, Inc.) o el Nellcor Puritan Bennet Achieva $\mathrm{PSO}_{2}$, ambos diseñados para proporcionar ventilación no invasiva en la UCI.

Desempeño comparativo. La Tabla 1 resume las principales diferencias entre estos ventiladores y los ventiladores convencionales. Gran parte de los ensayos clínicos de VNI en la insuficiencia respiratoria aguda han utilizado ventiladores portátiles ${ }^{13}$, y no existen estudios que hayan comparado la eficacia de estos ventiladores respecto de los ventiladores convencionales. Los datos existentes sugieren, en todo caso, que el alto estándar tecnológico de los ventiladores convencionales puede ser necesario en una pequeña proporción de pacientes con insuficiencia respiratoria grave que requieren manejo en la UCI. Esto explica que cuando la VNI se aplica en estas unidades predomine el uso de ventiladores convencionales ${ }^{14}$. En estos pacientes de mayor gravedad la sincronía entre paciente y ventilador probablemente juega un rol mucho más relevante y requiere la capacidad de monitorización y alarmas de los ventiladores convencionales ${ }^{15}$.

\section{Características técnicas de los ventiladores}

En esta sección sólo se hará mención a las características de los ventiladores portátiles, pues la descripción de los ventiladores convencionales escapa al objetivo de este documento. Sólo se describirá los mecanismos de control, que incluyen la variable controlada y las variables de fase. Las modalidades se detallan en otra sección. En la Tabla 2 se describen las características técnicas de algunos ventiladores de uso frecuente en nuestro país en unidades de cuidados intermedios, intensivos o en el domicilio. Nótese que algunos modelos, aún en uso, no han sido incluidos porque su fabricación ha sido discontinuada, como es el caso de los primeros equipos portátiles, fabricados por Respironics Inc., (BiPAP S/T y BiPAP S/T 30). Al momento de elegir un determinado modelo es conveniente tener en cuenta, dependiendo del lugar donde se utilizará, la simpleza de manipulación, el tamaño del equipo, el tamaño y tipo de información que proporciona la pantalla, el rango de presiones y

Tabla 1. Comparación entre ventiladores de flujo continuo y ventiladores convencionales

\begin{tabular}{lll}
\hline Características & $\begin{array}{l}\text { Flujo } \\
\text { continuo }\end{array}$ & $\begin{array}{l}\text { Conven- } \\
\text { cionales }\end{array}$ \\
\hline Modalidades & Limitadas & Todas \\
Monitorización & Limitada & Amplia \\
Alarmas & Limitadas & Todas \\
Problemas de disparo & Infrecuentes & Posibles \\
Compensación de fugas & Buena & Limitada \\
Mezclador de oxígeno & Excepcional & Siempre \\
Reinhalación de $\mathrm{CO}_{2}$ & Posible & Nunca \\
Peso & Livianos & Pesados \\
Costos & Reducidos & Altos \\
\hline
\end{tabular}


flujos, y la gama de alarmas. En general, el acceso a las funciones de estos ventiladores portátiles es poco intuitivo y la pantalla de información suele ser pequeña, siendo una excepción notable la del Breas Vivo 40. Esto se debe a que no existe aún una normativa internacional que regule su fabricación ${ }^{16}$.

Variable controlada. Cualquier ventilador, para poder operar, debe hacerse con el control del sistema respiratorio, algo que logra adquiriendo el comando de alguna de las variables involucradas en el movimiento del sistema respiratorio, esto es, presión, volumen o flujo. Dependiendo de cual variable controle, el ventila- dor proporcionará una onda de presión, flujo o volumen que se mantendrá inalterada durante los ciclos respiratorios y que sólo dependerá de su programación, independientemente de cualquier cambio que puedan experimentar las propiedades mecánicas del sistema respiratorio.

Todos los ventiladores portátiles controlan el flujo. Como se observa en la Figura 2, el gas filtrado desde el ambiente entra al equipo y pasa a través de una válvula solenoide proporcional manipulada electromagnéticamente. La válvula permite ajustar la tasa de flujo necesaria para lograr la IPAP y EPAP prefijadas. Para esto cuenta con transductores de flujo y presión a la salida del equipo que envían señales de retroali-

Tabla 2. Ventajas y desventajas potenciales de las diferentes interfaces

\begin{tabular}{|c|c|c|}
\hline Interface & Ventajas & Desventajas \\
\hline Nasal & $\begin{array}{l}\text { Bien tolerada en uso crónico } \\
\text { Bajo riesgo de aspiración } \\
\text { No produce claustrofobia }\end{array}$ & $\begin{array}{l}\text { Fugas bucales } \\
\text { Lesiones por apoyo del puente nasal }\end{array}$ \\
\hline Oro-nasal & $\begin{array}{l}\text { Buen control de fugas bucales } \\
\text { Útil en pacientes confusos }\end{array}$ & $\begin{array}{l}\text { Claustrofobia } \\
\text { Lesiones por apoyo del puente nasal } \\
\text { Riesgo de aspiración }\end{array}$ \\
\hline Facial total & $\begin{array}{l}\text { Espacio muerto funcional pequeño } \\
\text { Fugas menos probables } \\
\text { Útil en pacientes con claustrofobia } \\
\text { Útil cuando hay lesiones faciales/nasales } \\
\text { Útil en pacientes confusos }\end{array}$ & $\begin{array}{l}\text { Sufren grietas por estrés de material } \\
\text { Costo elevado }\end{array}$ \\
\hline Casco & $\begin{array}{l}\text { No tiene contacto con cráneo y cara } \\
\text { Confortable y bien tolerada }\end{array}$ & $\begin{array}{l}\text { Mayor reinhalación de } \mathrm{CO}_{2} \\
\text { Pueden requerir mayores presiones inspiratorias } \\
\text { Pueden aumentar trabajo inspiratorio por retardo } \\
\text { en el disparo }\end{array}$ \\
\hline
\end{tabular}

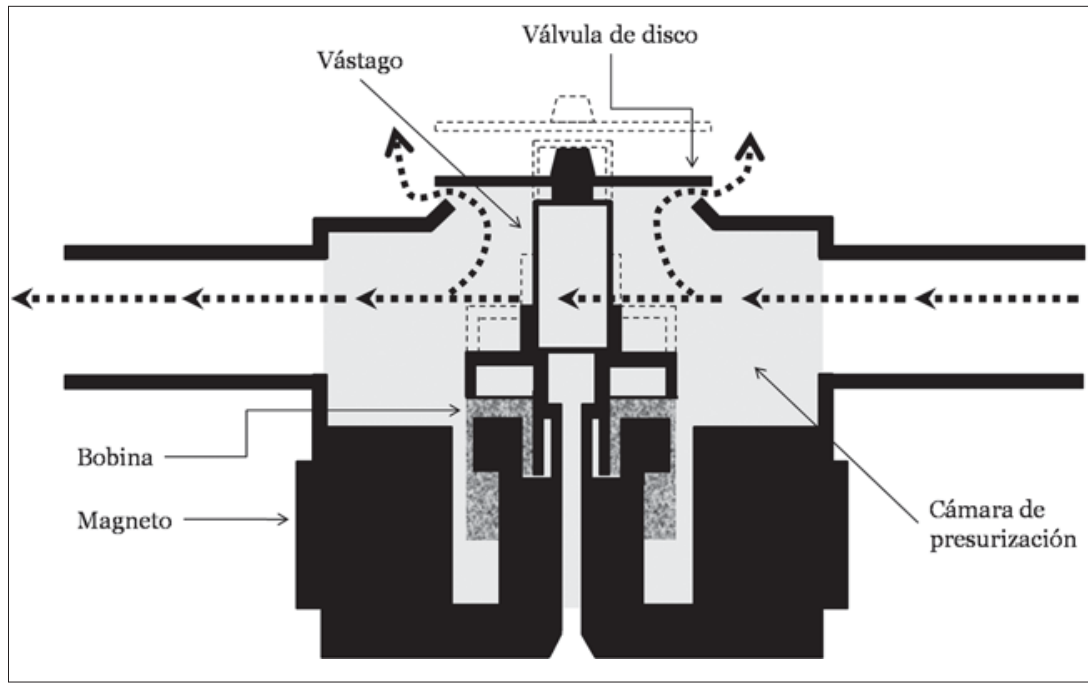

Figura 2. Válvula solenoide proporcional manipulada electromagnéticamente del ventilador Respironics BiPAP S/T-D. Para detalles ver texto. Modificada de Hess D R, Kacmarek R M. Noninvasive ventilation. En: Branson R D, Hess D R, Chatburn R L, eds. Respiratory care equipment. $2^{\text {nd }}$ Ed. Philadelphia: Lippincott Williams \& Willkins; 1999: 593-612. 


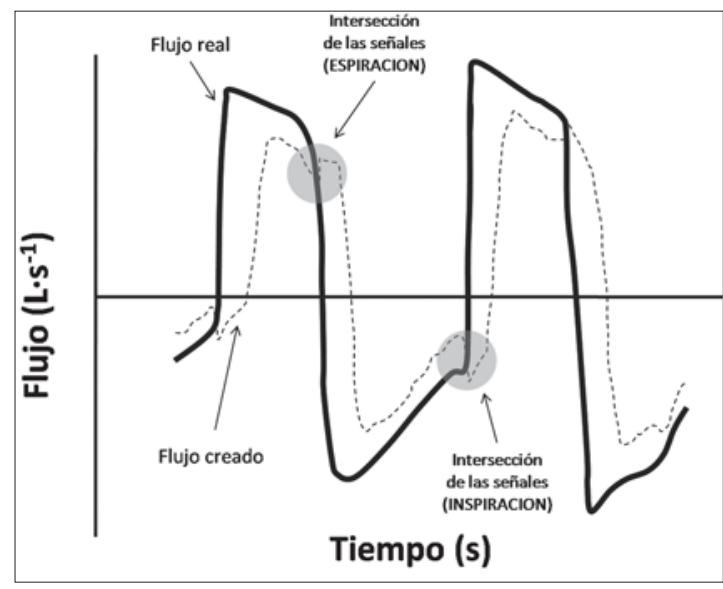

Figura 3. Sistema de gatillado y ciclado por la onda de flujo. Diseñado por Respironics, Inc. (Auto-Trak Sensitivity ${ }^{\mathrm{TM}}$ ), permite al ventilador generar una señal estimada del flujo del paciente (línea discontinua), pero desalineada en $0,25 \mathrm{~L} / \mathrm{s}$ y retrasada $300 \mathrm{~ms}$ de la onda de flujo real (línea continua). Tanto el gatillado como el ciclado a espiración se producen cuando ambas curvas se cruzan.

mentación a un microprocesador. La válvula está conformada por un electroimán, una bobina y un vástago unido a un disco. Como resultado de su acción, la cámara de presurización se abre o cierra al ambiente proporcionalmente a la fuerza del vástago que mueve al disco, dependiendo de la corriente eléctrica que atraviese la bobina. La magnitud de la corriente eléctrica es ajustada por el microprocesador.

Variables de fase. El ciclo respiratorio en el ventilador comienza al iniciarse la insuflación mecánica y finaliza con el inicio de la siguiente. Convencionalmente se le divide en cuatro fases: a) cambio de espiración a inspiración, que corresponde al gatillado; b) inspiración, en que es importante la velocidad de presurización y la variable limitante; c) cambio de inspiración a espiración, que corresponde al ciclado del ventilador; y d) espiración, que en la ventilación no invasiva corresponde a la EPAP. Esta división convencional del ciclo respiratorio permite examinar cómo se gatilla o dispara una inspiración, cómo se sostiene o limita la inspiración y qué criterio determina cuándo la inspiración finaliza.

Gatillado. Para que el gatillado sea posible, el ventilador debe detectar una señal proveniente del paciente que indique el inicio de una inspiración. La excepción a esto es el gatillado por tiempo, en que el ventilador comienza la inspiración de acuerdo con una frecuencia respiratoria prefijada. En el primer caso, cuando el esfuerzo inspiratorio del paciente genera un cambio en la variable medida y ésta supera un cierto umbral prefijado llamado sensibilidad, se activa la inspiración. Los ventiladores portátiles son gatillados por flujo, aunque la mayoría tiene también la opción de ser gatillado por tiempo. Los modelos recientes permiten ajustar manualmente la sensibilidad del gatillado por flujo, mientras que ésta venía prefijada por el fabricante en los equipos antiguos. La sensibilidad también puede ajustarse automáticamente, mediante un algoritmo del ventilador que analiza la forma de la onda de flujo. Este es el caso de los equipos de Respironics, Inc., que mediante software generan una señal estimada del flujo del paciente, pero desalineada en $0,25 \mathrm{~L} / \mathrm{seg}$ y retrasada $300 \mathrm{~ms}$ (AutoTrak Sensitivity $\left.{ }^{\mathrm{TM}}\right)^{17}$. El disparo se produce cuando ambas curvas se cruzan, como se observa en la Figura 3. Este tipo de gatillado reduce los esfuerzos inspiratorios perdidos (aquellos esfuerzos del paciente que no logran gatillar el ventilador) y disminuye el retardo del disparo (lapso de tiempo entre el inicio del esfuerzo del paciente y el inicio de la inspiración mecánica), pero puede propiciar auto-gatillado.

Variable limitante. La variable limitante es aquella que sostiene la inspiración. La inspiración mecánica se define como el intervalo que se extiende desde el inicio de la insuflación mecánica hasta el inicio de la espiración. En este período presión, flujo y volumen aumentan sus valores por sobre los basales, independientemente de qué variable controle el ventilador. Si una de ellas sólo aumenta hasta un valor prefijado significa que la modalidad ventilatoria está limitada por dicha variable. Es importante hacer notar, en todo caso, que si bien esta variable sostiene la inspiración, no determina cuándo termina el ciclo inspiratorio. Los ventiladores portátiles están limitados por presión, lo que significa que el operador programa un valor de presión y éste se mantiene constante mientras dure la inspiración.

Presurización. La pendiente de presurización, llamada también rampa o rise time, corresponde en la práctica al tiempo que transcurre desde el inicio de la inspiración mecánica hasta que se alcanza la presión inspiratoria prefijada (Figura 4). Debido a que la demanda ventilatoria es muy elevada en la fase inicial de la insuficiencia respiratoria aguda, cualquiera sea su causa, se requiere usualmente de una rápida presurización del sistema respiratorio ${ }^{18-20}$. Un tiempo de presurización muy lento o excesivamente rápido puede 


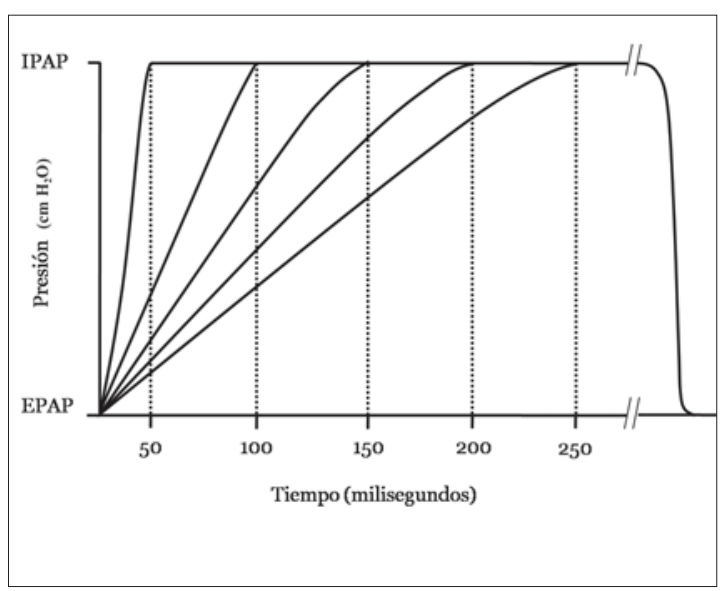

Figura 4. Pendiente, rampa o tiempo de presurización (rise time). Corresponde al tiempo, expresado en segundos o milisegundos, que transcurre desde el inicio de la inspiración mecánica hasta que se alcanza la presión inspiratoria prefijada. En la figura se describen cinco tiempos de presurización: 50, 100, 150, 200 y 250 ms.

aumentar el trabajo respiratorio ${ }^{19}$. En la etapa inicial el tiempo de presurización sugerido es de 50 a $150 \mathrm{~ms}$. A medida que se produce la estabilización, la demanda ventilatoria disminuye y puede ser necesario disminuirlo a 200-400 ms. El panel de los equipos portátiles tiene valores numéricos arbitrarios en lugar de milisegundos para programar esta variable, y suele variar entre 5 y 9 niveles.

Ciclado. Esta variable es la que determina el final de la inspiración una vez que ha alcanzado un valor prefijado. Cuando el ciclado es por presión, el ventilador mantiene el flujo de gas hasta que se alcanza una presión prefijada; en ese momento el flujo inspiratorio cesa y comienza la espiración. Si el ciclado es por volumen, el ventilador proporciona flujo hasta que se alcanza el volumen prefijado, iniciándose entonces la espiración. En los ventiladores portátiles el ciclado es por flujo, por lo que la inspiración finaliza cuando se alcanza un flujo umbral. El momento en que el ventilador debe iniciar la espiración está predeterminado de fábrica en los ventiladores portátiles antiguos, siendo uno de los criterios más empleados la reducción del flujo inspiratorio a un $25 \%$ del flujo máximo. Los nuevos equipos permiten modificar este parámetro, característica crucial para lograr una adecuada sincronía paciente-ventilador en ciertas patologías.

Para entender cómo programar el inicio de la espiración es necesario conocer previamente de qué forma las propiedades mecánicas del sistema respiratorio influyen en el ciclado ${ }^{21}$. Para ello analizaremos un patrón restrictivo, aplicable a los pacientes con distintas causas de insuficiencia respiratoria hipoxémica (edema pulmonar agudo, síndrome de distrés respiratorio agudo, neumonía), y un patrón obstructivo, principalmente relacionado a la enfermedad pulmonar obstructiva crónica (EPOC). En el primer caso, una vez que se alcanza el flujo máximo su descenso es muy rápido debido a la elevada retracción elástica del sistema respiratorio (Figura 5). Por esta razón, el criterio usual de inicio de la espiración (caída del flujo a un 25\% del máximo) se alcanza muy precozmente y la espiración mecánica comienza prematuramente. La consecuencia es

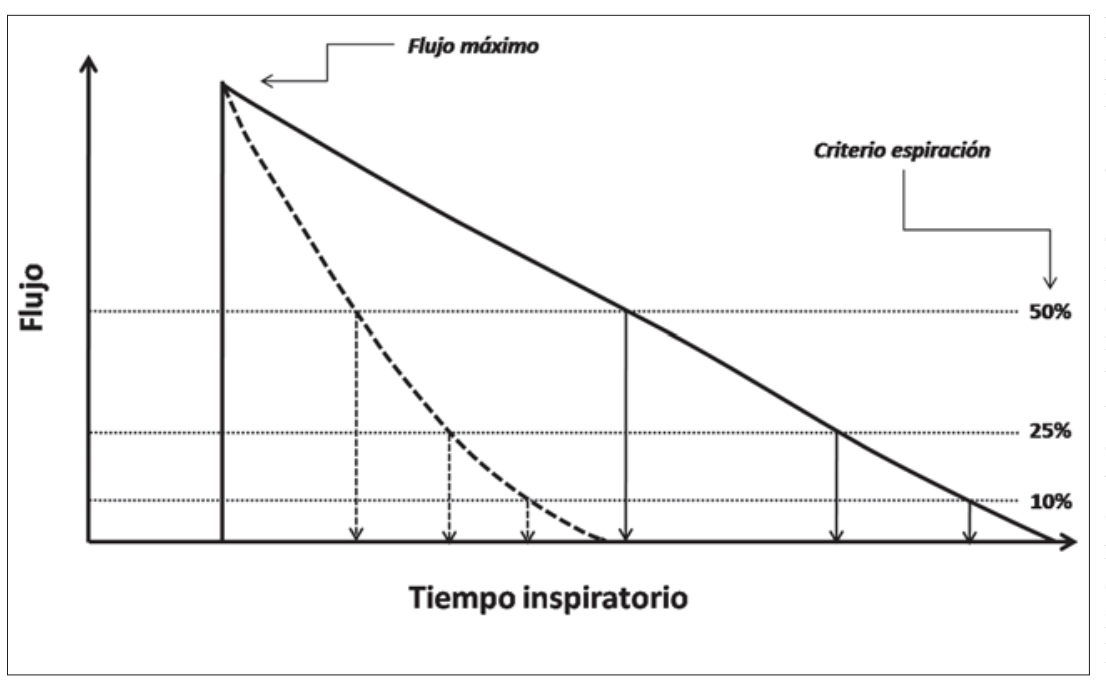

Figura 5. Efecto de las propiedades mecánicas del sistema respiratorio sobre el ciclado a espiración durante la ventilación con presión de soporte. Se describen tres criterios de ciclado: 50,25 y $10 \%$ del flujo máximo (líneas horizontales discontinuas); y dos condiciones clínicas: un paciente con una enfermedad pulmonar restrictiva aguda (curva de flujo discontinua) y un paciente con una enfermedad pulmonar obstructiva crónica (curva de flujo continua). Las flechas indican el momento en que termina la inspiración. Ver detalles en el texto. 
un VT pequeño, y por lo tanto insuficiente, una frecuencia respiratoria elevada, que trata de mantener un volumen minuto estable, y disnea. En el paciente descrito en la Figura 5, se aprecia que un criterio de ciclado de $10 \%$ del flujo máximo permite un tiempo inspiratorio mayor y, consecuentemente, un mayor VT. En el paciente con EPOC, una vez que se alcanza el flujo máximo su descenso es muy lento, debido a que el vaciamiento pulmonar está enlentecido por una combinación de obstrucción de la vía aérea pequeña y disminución de la retracción elástica. En este caso el criterio usual de inicio de la espiración (25\% del flujo máximo) se alcanza tardíamente. Como resultado, la inspiración mecánica se prolonga inapropiadamente. El paciente intenta espirar prematuramente, aumentando su trabajo respiratorio. Además, se produce atrapamiento aéreo ${ }^{22}$, aumenta la presión positiva intrínseca de final de espiración (PEEPi), y el paciente puede ser incapaz de gatillar la siguiente inspiración, lo que se conoce como esfuerzo inspiratorio perdido o malgastado ${ }^{23}$, o requerir un mayor esfuerzo inspiratorio para lograr el gatillado, lo que aumenta adicionalmente el trabajo respiratorio ${ }^{24}$. En la figura se observa que un criterio de ciclado más alto (50\% o mayor) permite un tiempo inspiratorio menor y evita los problemas mencionados.

Los criterios de ciclado son muy variables entre un paciente y otro, por lo que no pueden usarse reglas fijas para programar los ventilado- res. En general, los valores apropiados son aquellos que se asocian a la menor frecuencia respiratoria (idealmente menor de 25 respiraciones/ min) y al mayor VT. Cuando se selecciona un criterio apropiado el paciente se acoplará mejor al ventilador, lo que se traducirá en una mayor sensación de confort. Objetivamente, esto último debe traducirse en una reducción de las asincronías, como es el caso de los esfuerzos inspiratorios perdidos y la espiración prematura, efectos más fáciles de apreciar por un operador experimentado, ya sea mediante la cuidadosa observación del paciente o de las curvas de flujo y presión del ventilador.

Modalidades. Las modalidades empleadas en la VNI serán descritas en detalle en otra sección. Baste decir que la modalidad más frecuentemente empleada es la llamada binivel, que corresponde a la presión de soporte de los ventiladores convencionales. Esta es una modalidad limitada por presión y ciclada por flujo.

\section{Equipos y sincronía paciente-ventilador}

Cuando se juzga el desempeño de un ventilador para proporcionar VNI, no solamente interesa la capacidad para compensar fugas. Otro aspecto importante es la versatilidad que posea para ajustar las variables de fase y la forma en que estas responden a los requerimientos del

Tabla 3. Características de los ventiladores portátiles de flujo continuo para aplicación de ventilación no invasiva

\begin{tabular}{|c|c|c|c|c|c|c|c|}
\hline $\begin{array}{l}\text { Ventiladores } \\
\text { portátiles }\end{array}$ & $\begin{array}{c}\text { Synchrony } \\
\text { S/T }\end{array}$ & Harmony & Vivo 40 & Vivo 30 & $\begin{array}{c}\text { VPAP III } \\
\text { ST-A }\end{array}$ & $\begin{array}{c}\text { GoodKnight } \\
425 \text { ST }\end{array}$ & $\begin{array}{c}\text { KnightStar } \\
330\end{array}$ \\
\hline Fabricante & $\begin{array}{c}\text { Philips } \\
\text { Respironics }\end{array}$ & $\begin{array}{c}\text { Philips } \\
\text { Respironics }\end{array}$ & Breas & Breas & ResMed & $\begin{array}{l}\text { Puritan } \\
\text { Bennett }\end{array}$ & $\begin{array}{l}\text { Puritan } \\
\text { Bennett }\end{array}$ \\
\hline Modo asistido & $\mathrm{Si}$ & $\mathrm{Si}$ & $\mathrm{Si}$ & $\mathrm{Si}$ & $\mathrm{Si}$ & $\mathrm{Si}$ & $\mathrm{Si}$ \\
\hline Frecuencia & $0-30$ & $0-30$ & $4-40$ & $4-40$ & $5-30$ & $4-25$ & $3-30$ \\
\hline IPAP $\mathrm{cmH}_{2} \mathrm{O}$ & $4-30$ & $4-30$ & $4-30$ & $4-30$ & $2-30$ & $3-25$ & $3-30$ \\
\hline EPAP $\mathrm{cmH}_{2} \mathrm{O}$ & $4-25$ & $4-25$ & $2-30$ & $2-30$ & $2-25$ & $3-20$ & $3-20$ \\
\hline Rise Time & $\begin{array}{c}100-600 \mathrm{~ms} \\
6 \text { niveles }\end{array}$ & $\begin{array}{c}100-600 \mathrm{~ms} \\
6 \text { niveles }\end{array}$ & $1-9$ & $1-9$ & $150-900 \mathrm{~ms}$ & $\begin{array}{c}300-600 \mathrm{~ms} \\
5 \text { niveles }\end{array}$ & $0,5-3$ \\
\hline $\begin{array}{l}\text { Sensibilidad } \\
\text { inspiratoria }\end{array}$ & automática & automática & $1-9$ & $1-9$ & $1-3$ & $1-10$ & $1-5$ \\
\hline $\begin{array}{l}\text { Sensibilidad } \\
\text { espiratoria }\end{array}$ & automática & automática & 9 niveles & 3 niveles & $\begin{array}{c}1-3 \\
18-25-33 \%\end{array}$ & $1-10$ & $\begin{array}{c}1-5 \\
18-25- \\
32-44-55 \%\end{array}$ \\
\hline Humidificador & Opcional & Opcional & Opcional & Opcional & Integrado & Opcional & Opcional \\
\hline Batería & No & No & Incorporada & No & & Opcional & \\
\hline
\end{tabular}


paciente. Esto se debe a que el manejo apropiado de las fugas y de las variables de fase promueve una adecuada sincronía entre paciente y ventilador. El efecto de la sincronía pacienteventilador sobre los resultados de la ventilación no invasiva no ha sido evaluado. Sin embargo, un estudio realizado en 62 pacientes ventilados invasivamente mostró que la respiración asincrónica se asociaba con una estadía más prolongada en el ventilador ${ }^{25}$.

Clasificación de las asincronías. Existen distintas formas de clasificar las asincronías, siendo la más simple la que considera el momento del ciclo respiratorio en que se produce ${ }^{26}$. De acuerdo a este criterio, existen los siguientes tipos: a) asincronías del inicio del ciclo, esto es del gatillado; b) asincronías de la fase inspiratoria, también llamadas asincronías de flujo; y c) asincronías de ciclado, es decir, del inicio de la espiración ${ }^{26,27}$.

Desempeño comparativo. Un estudio reciente comparó 22 equipos, que incluían ventiladores convencionales de diferente antigüedad y equipos portátiles ${ }^{28}$. Al evaluar sensibilidad inspiratoria, retardo en el disparo y velocidad de presurización, los equipos portátiles tuvieron un desempeño superior a los ventiladores convencionales antiguos, pero similar al de los convencionales modernos. Sin embargo, se han observado notorias diferencias en el desempeño de los diferentes equipos portátiles ${ }^{4,29}$. Un estudio de laboratorio comparó 10 modelos recientes (Respironics, ResMed, Nellcor Puritan Bennet, Breas y Airox, entre otros $)^{29}$. Se simuló un patrón obstructivo y restrictivo, y niveles variables de esfuerzo inspiratorio, en presencia o no de fugas. Se observó que la sensibilidad inspiratoria era aceptable en todos los equipos. Sin embargo, el retardo del disparo fue inferior a $100 \mathrm{~ms}$ sólo en la mitad de ellos y si bien fue siempre menor a $200 \mathrm{~ms}$, debe tenerse en cuenta que valores superiores a $150 \mathrm{~ms}$ pueden ser percibidos por los pacientes ${ }^{30}$. La velocidad de presurización fue altamente variable, reduciéndose proporcionalmente a la magnitud del esfuerzo inspiratorio. La capacidad de detectar el inicio de la espiración empleando los valores por defecto de los diferentes modelos mostró retardo en el ciclado en condiciones simuladas de obstrucción bronquial y ciclado prematuro frente a una condición restrictiva, situación que pudo solucionarse ajustando este parámetro en aquellos equipos que lo permitían.

\section{Interfaces}

Se denomina interface al dispositivo que conecta al paciente al ventilador. La elección de una apropiada interface resulta intuitivamente crítica para el éxito de la VNI, en la medida que el grado de confort que ésta proporcione favorecerá la aceptación del procedimiento por parte del paciente ${ }^{31}$.

Tipos de interface. En el caso de la VNI, las mascarillas son las interfaces de preferencia, que pueden ser nasales u oro-nasales. Asimismo, existen las piezas bucales, las almohadillas nasales, las máscaras faciales totales y los cascos.

Las mascarillas están compuestas de una armazón de plástico rígido y transparente, bordeada por una almohadilla de silicona o gel, que favorece un sellado hermético entre la mascarilla y la piel. Pueden o no tener orificios de fuga, destinadas a la evacuación del gas espirado y a evitar la reinhalación de $\mathrm{CO}_{2}$ (Figura 6). Las mascarillas con orificio de fuga están diseñadas específicamente para ser usadas con ventiladores portátiles de flujo continuo. Por su parte, las

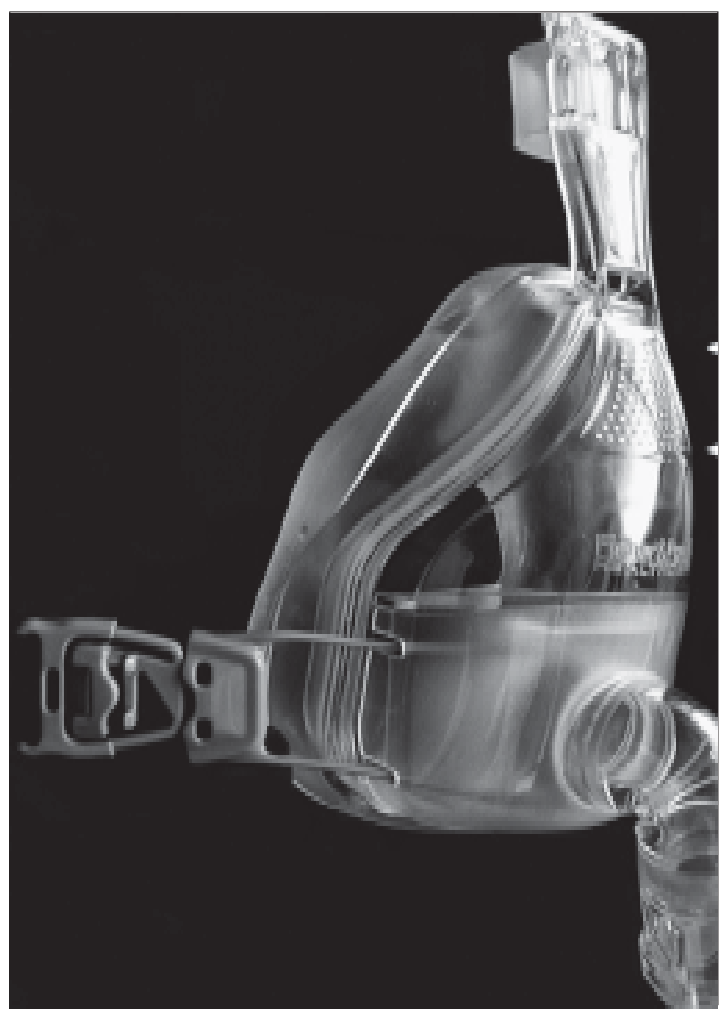

Figura 6. Mascarilla oro-nasal (Fisher \& Paykel Healthcare Corporation Limited). Las flechas muestran los orificios de fuga intencional que reducen el riesgo de reinhalación de $\mathrm{CO}_{2}$. 


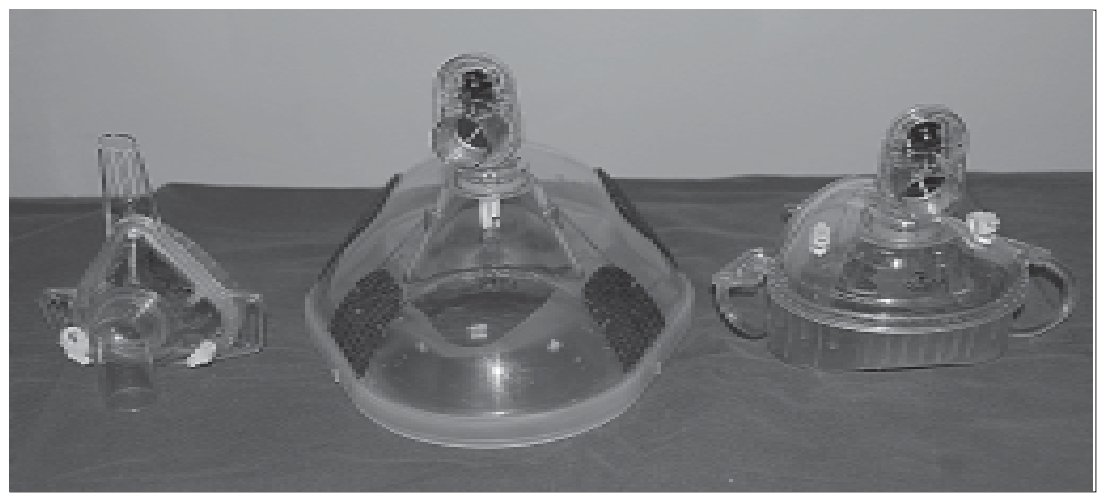

Figura 7. Tres tipos de interface frecuentemente empleadas en la ventilación no invasiva: mascarilla nasal, a la izquierda; máscara facial total, al centro; y mascarilla oro-nasal, a la derecha. (Philips Respironics, Inc.).

mascarillas sin fugas intencionales son útiles para emplearse con ventiladores convencionales; si se emplean con ventiladores portátiles es necesario conectar un adaptador fenestrado al circuito, cercano a la interface, con el fin de evitar la recirculación de $\mathrm{CO}_{2}$. Todas las mascarillas se conectan a la cara mediante arneses de fijación, generalmente de cuatro lengüetas, dos superiores y dos inferiores.

Las principales características de las interfaces usadas en la insuficiencia respiratoria aguda se describen a continuación, mientras que un resumen de sus ventajas y desventajas se proporciona en la Tabla 2. Asimismo, la Figura 7 muestra tres de estas interfaces. En el caso de la VNI, las mascarillas son las interfaces de preferencia, que pueden ser nasales u oro-nasales. Asimismo, existen las piezas bucales, las almohadillas nasales, las máscaras faciales totales y los cascos.

Mascarillas nasales. Sólo cubren la nariz, apoyándose sobre el puente nasal, los pómulos y el labio superior. La mayoría se fabrica con polivinilo y algunos modelos emplean una almohadilla o gel de silicona para disminuir la presión sobre los puntos de apoyo en la cara.

Mascarillas oro-nasales. Estas mascarillas se apoyan sobre el tabique nasal, los pómulos y el labio inferior o el mentón, reduciendo las fugas bucales que son muy frecuentes en la insuficiencia respiratoria aguda. Las mascarillas oronasales requieren de una válvula de seguridad anti asfixia que se abra al ambiente en caso de falla del ventilador (Figura 8). Estas válvulas suelen abrirse también cuando el flujo inspiratorio es insuficiente para la demanda del paciente, cuando la presión positiva espiratoria es excesivamente baja, y cuando se emplean con ventiladores convencionales en que el flujo es discontinuo.

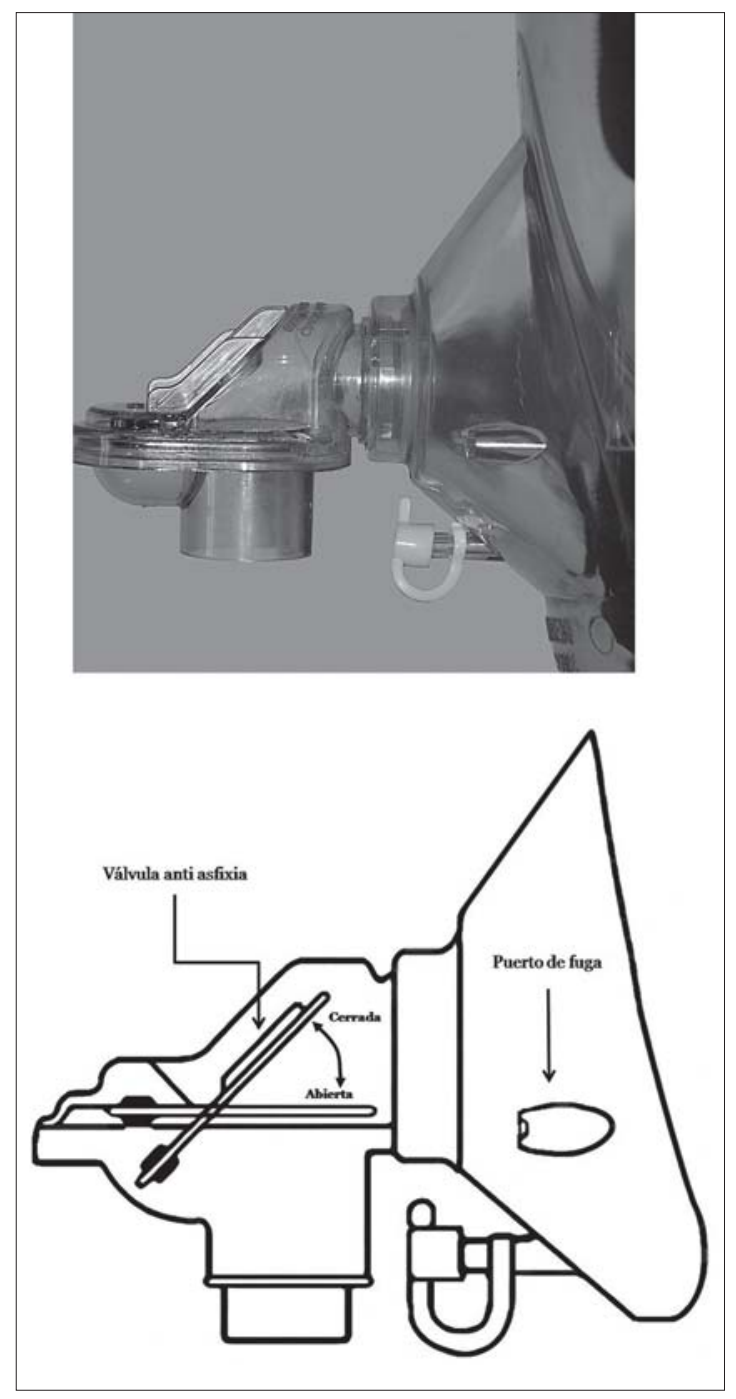

Figura 8. Detalle de una válvula anti asfixia, que se abre al ambiente cuando la presión del sistema desciende a valores inferiores a $3 \mathrm{~cm} \mathrm{H}_{2} \mathrm{O}$ por una falla del ventilador. Estas válvulas se emplean usualmente en las mascarillas oro-nasales y en las máscaras faciales totales. Nótese que el orificio de fuga de esta máscara tiene un diseño diferente al modelo de la figura 6. 
Máscara facial total. La máscara facial total (Total Face Mask ${ }^{\mathrm{TM}}$, Philips Respironics Inc.) cubre todo el rostro y es sostenida por un sistema de arneses laterales de muy fácil instalación. Existen en un solo tamaño y son reutilizables. Al igual que las mascarillas oro-nasales cuentan con una válvula anti asfixia y poseen orificios de fuga intencional. Dado que su superficie de plástico transparente suele agrietarse con facilidad, Philips Respironics liberará pronto al mercado una versión más resistente (Figura 9).

Cascos. El casco (Helmet Costar®, Starmed, Italia) está constituido por un cilindro de polivinilo transparente, libre de látex. La fijación se mantiene mediante un anillo rígido y fijo en la parte inferior y por dos arneses cruzados sujetos a este anillo rígido por delante y detrás. La parte inferior del anillo está rodeada por una membrana transparente y laxa que cubre el cuello en forma de collar. El circuito del ventilador se adosa a una conexión lateral (Figura 10).

Desempeño comparativo. Una revisión de los estudios publicados hasta el año $2004^{31}$, mostró que en la insuficiencia respiratoria aguda se utiliza de preferencia mascarillas oro-nasales (63\%), seguidas por las mascarillas nasales $(31 \%)$. Aunque las dos tienen efectos similares sobre el intercambio de gases, las máscaras nasales son peor toleradas en este escenario ${ }^{32}$. Por el contrario, en la insuficiencia respiratoria crónica, las máscaras nasales son las más comúnmente usadas $(73 \%)^{1,31}$, y las mejor toleradas, aunque exhiben una eficacia levemente inferior que las máscaras oro-nasales para reducir la $\mathrm{PaCO}_{2}{ }^{33}$. Esto es particularmente importante durante la aplicación nocturna de la VNI, debido a fugas bucales, que pueden interferir además con la calidad del sueño ${ }^{34}$. El uso de cascos se ha descrito sólo recientemente en la insuficiencia respiratoria aguda. Su ventaja potencial es que no provocan lesiones faciales y son bien tolerados, pero se asocian a mayor reinhalación de $\mathrm{CO}_{2}{ }^{35}$, a la vez que pueden retardar el disparo y afectar la velocidad de presurización ${ }^{36}$. La reinhalación puede representar un problema importante en la insuficiencia respiratoria aguda hipercápnica de la EPOC, pues se ha observado que la corrección de la hipercapnia es más lenta con el casco, particularmente en los pacientes que fraca$\operatorname{san}^{35}$. Si bien estos efectos adversos pueden corregirse aumentando los niveles de presión inspiratoria y de $\mathrm{PEEP}^{36}$, debe tenerse en cuenta que su empleo requiere una cuidadosa monitorización y ajuste del ventilador ${ }^{37}$.

Complicaciones de las interfaces. El uso de interfaces se asocia a una serie de complicaciones. Las complicaciones más frecuentes son las fugas de aire, que experimenta el $100 \%$ de los pacientes en algún momento de su uso; la congestión y desecamiento nasal (25-70\%); la irritación ocular (15-30\%); el eritema nasal (10$20 \%)$; la ulceración nasal (5-10\%); y la intolerancia a la mascarilla por falta de confort (50\%).

Como se ve, la complicación más importante corresponde a fugas no intencionales. Cuando se emplean mascarillas nasales, las fugas bucales ocasionan un aumento compensatorio del flujo
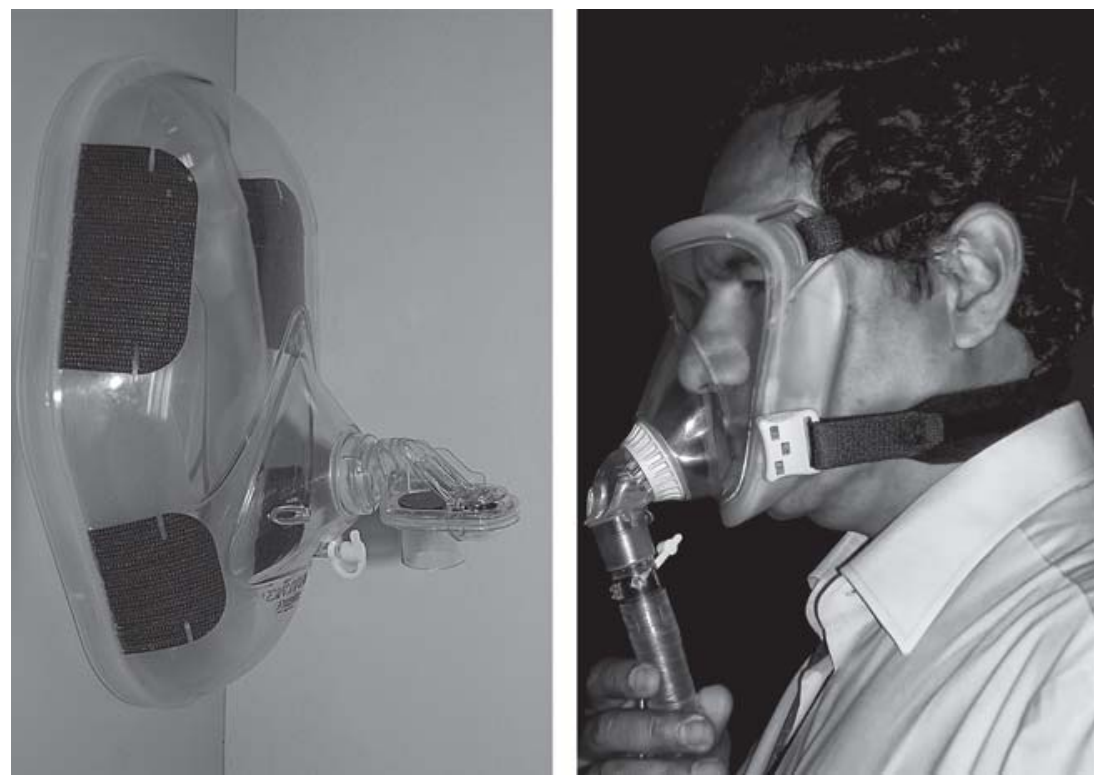

Figura 9. Máscaras faciales totales de Philips Respironics, Inc. El nuevo modelo, más resistente, es el de la derecha (cortesía Dr. César Maquilón). 


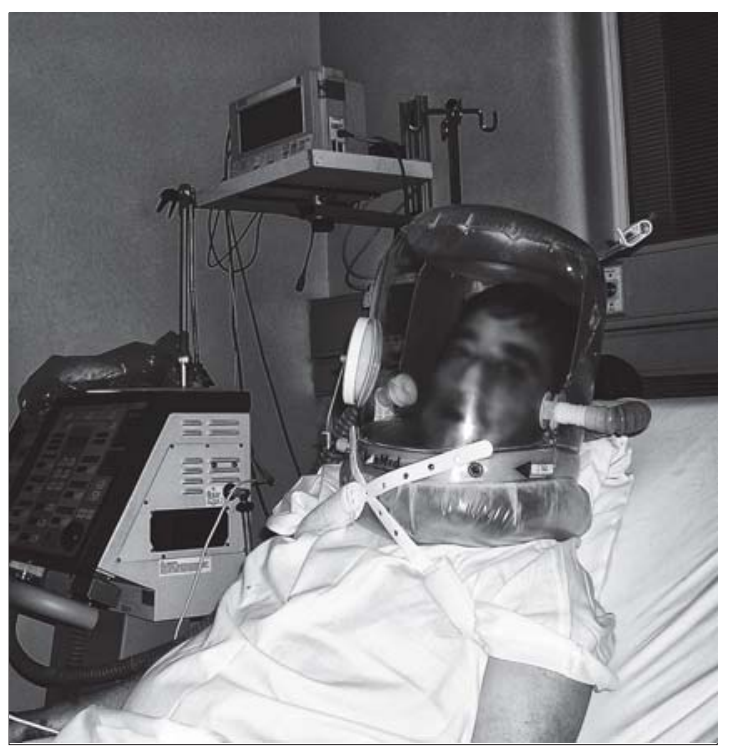

Figura 10. Casco (Helmet CaStar®; StarMed, Italia).

por parte del ventilador y provocan consecuentemente congestión y desecación nasal. Las fugas hacia los ojos, por su parte, provocan congestión e irritación ocular. La presencia de fugas se debe generalmente a la selección inadecuada de la interface; si este error no se reconoce, puede conducir al personal a cargo a aumentar la presión de sujeción de la mascarilla, provocando lesiones por apoyo, generalmente sobre el puente nasal.

Otras complicaciones se deben al empleo de presiones inspiratorias excesivas, las que pueden por un lado acentuar las fugas, y por otro, provocar distensión abdominal, dolores naso sinusales y otalgia, y en casos extremos neumotórax.

\section{Humidificación}

La humidificación normalmente no es necesaria durante la VNI en pacientes con insuficiencia respiratoria aguda. El uso de humidificadores se recomienda durante la aplicación domiciliaria de VNI a pacientes con insuficiencia respiratoria crónica, si bien su utilidad se ha inferido de estudios en pacientes con apnea del sueño ${ }^{1}$. El problema suele ser frecuente al emplear mascarillas nasales, ya que éstas promueven las fugas bucales y por lo tanto altos flujos nasales unidireccionales. El flujo unidireccional seca la mucosa nasal, promueve la liberación de mediadores de la inflamación e incrementa la resistencia nasal ${ }^{38}$. La humidificación calefaccio- nada aumenta la humedad relativa de la vía aérea, reduce la resistencia nasal y podría aumentar la adherencia al uso del ventilador ${ }^{38-40}$. Se recomienda el empleo de humidificadores calefaccionados en lugar de narices humidificadoras, ya que estas pueden afectar la resistencia de los circuitos, aumentando el trabajo inspiratorio.

\section{Bibliografía}

1.- SCHONHOFER B, SORTOR-LEGER S. Equipment needs for noninvasive mechanical ventilation. Eur Respir J 2002; 20: 1029-36.

2.- VIGNAUX L, TASSAUX D, JOLLIET P. Performance of noninvasive ventilation modes on ICU ventilators during pressure support: a bench model study. Intensive Care Med 2007; 33: 1444-51.

3.- GIRAULT C, RICHARD J C, CHEVRON V, TAMION F, PASQUIS P, LEROY J, et al. Comparative physiologic effects of noninvasive assist-control and pressure support ventilation in acute hypercapnic respiratory failure. Chest 1997; 111: 1639-48.

4.- HIGHCOCK M P, SHNEERSON J M, SMITH I E. Functional differences in bi-level pressure preset ventilators. Eur Respir J 2001; 17: 268-73.

5.- MEHTA S, MCCOOL F D, HILL N S. Leak compensation in positive pressure ventilators: a lung model study. Eur Respir J 2001; 17: 259-67.

6.- MIYOSHI E, FUJINO Y, UCHIYAMA A, MASHIMO T, NISHIMURA M. Effects of gas leak on triggering function, humidification, and inspiratory oxygen fraction during noninvasive positive airway pressure ventilation. Chest 2005; 128: 3691-8.

7.- FERGUSON G T, GILMARTIN M. $\mathrm{CO}_{2}$ rebreathing during BiPAP ventilatory assistance. Am J Respir Crit Care Med 1995; 151: 1126-35.

8.- LOFASO F, BROCHARD L, TOUCHARD D, HANG T, HARF A, ISABEY D. Evaluation of carbon dioxide rebreathing during pressure support ventilation with airway management system (BiPAP) devices. Chest 1995; 108: 772-8.

9.- SAATCI E, MILLER D M, STELL I M, LEE K C, MOXHAM J. Dynamic dead space in face masks used with noninvasive ventilators: a lung model study. Eur Respir J 2004; 23: 129-35.

10.- HILL N S, CARLISLE C, KRAMER N R. Effect of a nonrebreathing exhalation valve on long-term nasal ventilation using a bilevel device. Chest 2002; 122 : 84-91.

11.- THYS F, LIISTRO G, DOZIN O, MARION E, RODENSTEIN D O. Determinants of $\mathrm{FIO}_{2}$ with oxygen supplementation during noninvasive two-level positive pressure ventilation. Eur Respir J 2002; 19: 653-7.

12.- SCHWARTZ A, KACMAREK R, HESS D. Factors affecting oxygen delivery with bi-level positive airway pressure. Respir Care 2004; 49: 270-5.

13.- COMMITTEE. BTS SOCIETY. Non-invasive ventilation in acute respiratory failure. Thorax 2002; 57: $192-211$.

14.- CARLUCCI A, RICHARD J-C, WYSOCKI M, LEPAGE E, BROCHARD L. Noninvasive versus conventional mechanical ventilation. An epidemiologic survey. Am J Respir Crit Care Med 2001; 163: 874-80.

15.- KACMAREK R M. NIPPV: patient-ventilator 
synchrony, the difference between success and failure? Intensive Care Medicine 1999; 25: 645-7.

16.- GONZÁLEZ-BERMEJO J, LAPLANCHE V, HUSSEINI F E, DUGUET A, DERENNE J P, SIMILOWSKI T. Evaluation of the user-friendliness of 11 home mechanical ventilators. Eur Respir J 2006; 27: 1236-43.

17.- PRINIANAKIS G, KONDILI E, GEORGOPOULOS D. Effects of the flow waveform method of triggering and cycling on patient-ventilator interaction during pressure support. Intensive Care Medicine 2003; 29: 1950-9.

18.- BONMARCHAND G, CHEVRON V, CHOPIN C, JUSSERAND D, GIRAULT C, MORITZ F, et al. Increased initial flow rate reduces inspiratory work of breathing during pressure support ventilation in patients with exacerbation of chronic obstructive pulmonary disease. Intensive Care Med 1996; 22: 1147-54.

19.- CHIUMELLO D, PELOSI P, CROCI M, BIGATELLO L M, GATTINONI L. The effects of pressurization rate on breathing pattern, work of breathing, gas exchange and patient comfort in pressure support ventilation. Eur Respir J 2001; 18: 107-14.

20.- BONMARCHAND G, CHEVRON V, MÉNARD J, GIRAULT C, MORITZ-BERTHELOT F, PASQUIS P, et al. Effects of pressure ramp slope values on the work of breathing during pressure support ventilation in restrictive patients. Crit Care Med 1999; 27: 71522.

21.- HESS D. Ventilator waveforms and the physiology of pressure support ventilation. Respir Care 2005; 50 : 166-86.

22.- YOUNES M, KUN J, WEBSTER K, ROBERTS D. Response of ventilator-dependent patients to delayed opening of exhalation valve. Am J Respir Crit Care Med 2002; 166: 21-30.

23.- VASSILAKOPOULOS T. Understanding wasted/ ineffective efforts in mechanically ventilated COPD patients using the Campbell diagram. Intensive Care Medicine 2008; 34: 1336-9.

24.- TASSAUX D, GAINNIER M, BATTISTI A, JOLLIET P. Impact of expiratory trigger setting on delayed cycling and inspiratory muscle workload. Am J Respir Crit Care Med 2005; 172: 1283-9.

25.- THILlE A W, RODRÍGUEZ P, CABELLO B, LELLOUCHE F, BROCHARD L. Patient-ventilator asynchrony during assisted mechanical ventilation. Intensive Care Medicine. 2006; 32: 1515-22.

26.- NILSESTUEN J, HARGETT K. Using ventilator graphics to identify patient-ventilator asynchrony. Respir Care 2005; 50: 202-34.

27.- THILLE A W, BROCHARD L. Promoting patientventilator synchrony. Clin Pulm Med 2007; 14: 3509.

28.- RICHARD J C, CARLUCCI A, BRETON L, LANGLAIS N, JABER S, MAGGIORE S, et al. Bench testing of pressure support ventilation with three different generations of ventilators. Intensive Care Medicine 2002; 28: 1049-57.

29.- BATTISTI A, TASSAUX D, JANSSENS J-P, MICHOTTE J-B, JABER S, JOLLIET P. Performance characteristics of 10 home mechanical ventilators in pressure-support mode: A comparative bench study. Chest 2005; 127: 1784-92.

30.- WHITELAW W, DERENNE J, MILIC-EMILI J. Occlusion pressure as a measure of respiratory center output in conscious man. Respir Physiol 1975; 23: 181-99.

31.- ELLIOTT M W. The interface: crucial for successful noninvasive ventilation. Eur Respir J 2004; 23: 7-8.

32.- KWOK H, MCCORMACK J, CECE R, HOUTCHENS J, HILL N S. Controlled trial of oronasal versus nasal mask ventilation in the treatment of acute respiratory failure. Crit Care Med 2003; 31: 468-73.

33.- NAVALESI P, FANFULLA F, FRIGERIO P, GREGORETTI C, NAVA S. Physiologic evaluation of noninvasive mechanical ventilation delivered with three types of masks in patients with chronic hypercapnic respiratory failure. Crit Care Med 2000; 28 : 1785-90.

34.- TESCHLER H, STAMPA J, RAGETTE R, KONIETZKO N, BERTHON-JONES M. Effect of mouth leak on effectiveness of nasal bilevel ventilatory assistance and sleep architecture. Eur Respir J 1999; 14: 1251-17.

35.- ANTONELLI M, PENNISI M A, PELOSI P, GREGORETTI C, SQUADRONE V, ROCCO M, et al. Noninvasive positive pressure ventilation using a helmet in patients with acute exacerbation of chronic obstructive pulmonary disease: A feasibility study. Anesthesiology 2004; 100: 16-24.

36.- MOERER O, FISCHER S, HARTELT M, KUVAKI B, QUINTEL M, NEUMANN P. Influence of two different interfaces for noninvasive ventilation compared to invasive ventilation on the mechanical properties and performance of a respiratory system: A lung model study. Chest 2006; 129: 1424-31.

37.- CHIUMELLO D. Is the Helmet different than the face mask in delivering noninvasive ventilation? Chest 2006; 129: 1402-3.

38.- RICHARDS G, CISTULLI P, UNGAR R, BERTHONJONES M, SULLIVAN C. Mouth leak with nasal continuous positive airway pressure increases nasal airway resistance. Am J Respir Crit Care Med 1996; 154: 182-6.

39.- MARTINS DE ARAUJO M T, VIEIRA S B, VASQUEZ E C, FLEURY B. Heated humidification or face mask to prevent upper airway dryness during continuous positive airway pressure therapy. Chest 2000; 117 : 142-7.

40.- MASSIE C A, HART R W, PERALEZ K, RICHARDS $G$ N. Effects of humidification on nasal symptoms and compliance in sleep apnea patients using continuous positive airway pressure. Chest 1999; 116 : 403-8.

Correspondencia a:

Dr. Orlando Díaz P.

E-mail: odiazp@vtr.net 